\title{
A Further Study of Almost Periodic Time Scales with Some Notes and Applications
}

\author{
Chao Wang ${ }^{1}$ and Ravi P. Agarwal ${ }^{2,3}$ \\ ${ }^{1}$ Department of Mathematics, Yunnan University, Kunming, Yunnan 650091, China \\ ${ }^{2}$ Department of Mathematics, Texas A\&M University-Kingsville, Kingsville, TX 78363-8202, USA \\ ${ }^{3}$ Department of Mathematics, Faculty of Science, King Abdulaziz University, P.O. Box 80203, Jeddah 21589, Saudi Arabia
}

Correspondence should be addressed to Chao Wang; chaowang@ynu.edu.cn

Received 2 June 2014; Revised 10 July 2014; Accepted 13 July 2014; Published 7 August 2014

Academic Editor: Elena Braverman

Copyright (C) 2014 C. Wang and R. P. Agarwal. This is an open access article distributed under the Creative Commons Attribution License, which permits unrestricted use, distribution, and reproduction in any medium, provided the original work is properly cited.

\begin{abstract}
We introduce three equivalent concepts of almost periodic time scales as a further study of the corresponding concept proposed in Li and Wang (2011) and several examples of almost periodic time scales which are not periodic are provided. Furthermore, the concepts of almost periodic functions are redefined under the sense of this new timescale concept. Finally, almost periodicity of Cauchy matrix for dynamic equations is proved under these new definitions. Based on these results, the existence of almost periodic solutions to a class of nonlinear dynamic equations is investigated by the almost periodicity of Cauchy matrix on almost periodic time scales. Besides, as an application, we apply our results to a class of high-order Hopfield neural networks.
\end{abstract}

\section{Introduction}

Almost periodicity is a recent concept in the literature of time scales. It was formally introduced by Li and Wang in $[1,2]$, and based on this, some results concerning almost periodicity for dynamic equations on time scales were proved and a series of relative applications were published (see [3-6]). Meanwhile, some mathematicians are interested in this subject, and some relative works appeared (see [7-12]).

As everyone knows, the almost periodic time scales play a very important and fundamental role in redefining some classical functions on time scales such as almost periodic functions [1], pseudo almost periodic functions [6] and almost automorphic functions [7], and even weighted pseudo almost automorphic functions [3]. In [13], by using the concept and properties of almost periodic time scales, Lizama et al. prove a strong connection between almost periodic functions on time scales and almost periodic functions on $\mathbb{R}$ and then give an application to difference equations on $\mathbb{T}=h \mathbb{Z}$. Besides, some works have been done under the concept of almost periodic time scales; see $[7,13,14]$.

However, some mathematicians find that the concept of almost periodic time scales in [1] is exactly like the concept of periodic time scales in [15]. Furthermore, in Section 3 of [7], indeed, all invariant under translations time scales are periodic time scales, that is, from Example 3.9 to Example 3.11, which indicate that we investigated almost periodic problems of dynamic equations under the periodic time scales in the past, and all the obtained results are valid for all periodic time scales, particularly, for two special periodic time scales: $\mathbb{T}=\mathbb{R}$ and $\mathbb{T}=\mathbb{Z}$. Although this method can unify the continuous and discrete situations effectively, whether or not there exists a time scale which is almost periodic but not periodic if we introduce a new concept of almost periodic time scales. Therefore, it is very necessary to investigate the almost periodic time scales and introduce a more general and accurate definition that can strictly include all periodic time scales to overcome some difficulties in this research field.

It is known to all that the Cauchy matrix is very important in the research of dynamic equations. However, by using the almost periodicity of Cauchy matrix to discuss almost periodic problems of dynamic equations, we will encounter a problem. Let $W(t, s)$ be the Cauchy matrix of the following dynamic equations:

$$
x^{\Delta}=A(t) x(t)
$$


where $A \in C\left(\mathbb{T}, \mathbb{R}^{n \times n}\right)$ is an almost periodic matrix-valued function and $x \in C\left(\mathbb{T}, \mathbb{R}^{n}\right)$. $C(\mathbb{T}, \mathbb{X})$ denotes the set of all continuous functions from $\mathbb{T}$ to the Banach space $\mathbb{X}$. Consider the following nonlinear dynamic equations:

$$
x^{\Delta}=A(t) x(t)+f(t, x(t))
$$

where $A \in C\left(\mathbb{T}, \mathbb{R}^{n \times n}\right)$ is an almost periodic matrix-valued function and $x \in C\left(\mathbb{T}, \mathbb{R}^{n}\right)$, and $f \in C\left(\mathbb{T} \times \mathbb{R}^{n}, \mathbb{R}^{n}\right)$ is almost periodic in $t$ uniformly for $x \in \mathbb{R}^{n}$. By the Cauchy matrix of (1), in this paper, we can get a bounded solution of (2) as follows:

$$
x(t)=\int_{-\infty}^{t} W(t, \sigma(s)) f(s, x(s)) \Delta s,
$$

and the question then arises: for any $\varepsilon>0$, whether or not the $\varepsilon$-almost period $\tau$ of the matrix function $A(t)$ is valid such that the following inequality holds:

$$
\begin{gathered}
\|W(t+\tau, \sigma(s+\tau))-W(t, \sigma(s))\| \\
\leq \varepsilon \Gamma_{0} e_{\ominus \alpha}(t, \sigma(s)), \quad t \geq s,
\end{gathered}
$$

if the Cauchy matrix satisfies the inequality:

$$
\|W(t, s)\| \leq K e_{\ominus \alpha}(t, s), \quad t \geq s,
$$

where $\alpha, \Gamma_{0}, K$ are positive constants. As everyone knows, if $\mathbb{T}$ is a $\tau$-periodic time scale, then

$$
\sigma(t+\tau)=\sigma(t)+\tau
$$

so (4) will turn into

$$
\begin{gathered}
\|W(t+\tau, \sigma(s)+\tau)-W(t, \sigma(s))\| \\
\leq \varepsilon \Gamma_{0} e_{\ominus \alpha}(t, \sigma(s)), \quad t \geq s,
\end{gathered}
$$

which seems too special even though its validity can be shown on all periodic time scales, particularly on $\mathbb{T}=\mathbb{R}$ and $\mathbb{T}=\mathbb{Z}$. Nevertheless, if we can introduce a new concept of almost periodic time scales which strictly includes the periodic time scales such that (4), rather than (7), is valid under the condition (5), that is, the almost periodicity of Cauchy matrix can be guaranteed without considering (6) on this kind of general time scales, then the almost periodicity of (3) can easily be shown under (4).

Motivated by the above, the almost periodic time scales need a further study since the concept proposed in the past [1] is actually periodic, which will lead to some research difficulties and specificity of the obtained results. In this paper, we will introduce three equivalent concepts of almost periodic time scales as a revision of the corresponding concept proposed in [1], and several examples of almost periodic time scales which are not periodic are provided. Furthermore, the concepts of almost periodic functions are redefined under the sense of this new timescale concept.

The present paper is organized as follows. In Section 2, we will introduce three equivalent concepts of almost periodic time scales and give some key notes; then, the concepts of almost periodic functions are redefined under the sense of this new timescale definition. Furthermore, several examples of almost periodic time scales which are not periodic are provided. In Section 3, the almost periodicity of Cauchy matrix is analyzed under these new definitions; then, the almost periodicity of (3) is easily shown under the condition (5). In Section 4, our results are applied to investigate the existence of almost periodic solutions to a class of high-order Hopfield neural networks on time scales. In Section 5, we conduct a further discussion of almost periodic time scales, on which the concept of almost automorphic functions is introduced, and some relative works will appear in our future research.

It is worth noting that the three new equivalent definitions of almost periodic time scales proposed in this paper will play an important role in analyzing almost periodicity, pseudo almost periodicity, and weighted pseudo almost periodicity of Cauchy matrix for dynamic equations on time scales. All results obtained in [1] and their proof processes are valid under these new concepts without considering the set $\Pi$, which will be referred to in the next section.

\section{A Further Study of Almost Periodic Time Scales and Some Notes}

A time scale $\mathbb{V}$ is a closed subset of $\mathbb{R}$. It follows that the jump operators $\sigma, \rho: \mathbb{T} \rightarrow \mathbb{T}$ defined by $\sigma(t)=\inf \{s \in \mathbb{T}: s>t\}$ and $\rho(t)=\sup \{s \in \mathbb{T}: s<t\}$ (supplemented by inf $\emptyset:=$ $\sup \mathbb{\mathbb { T }}$ and $\sup \emptyset:=\inf \mathbb{\mathbb { }})$ are well defined. The point $t \in \mathbb{T}$ is left-dense, left-scattered, right-dense, and right-scattered if $\rho(t)=t, \rho(t)<t, \sigma(t)=t$, and $\sigma(t)>t$, respectively. If $\mathbb{T}$ has a right-scattered minimum $m$, define $\mathbb{T}_{k}:=\mathbb{T} \backslash m$; otherwise, set $\mathbb{T}^{k}=\mathbb{T}$. For the notations $[a, b]_{\mathbb{T}},[a, b)_{\mathbb{T}}$ and so on, we will denote time scale intervals

$$
[a, b]_{\mathbb{T}}=\{t \in \mathbb{T}: a \leq t \leq b\},
$$

where $a, b \in \mathbb{T}$ with $a<\rho(b)$. For more knowledge of time scales, one can see [15-18].

Firstly, we recall the concept of almost periodic time scales in [1].

Definition 1 (see [1]). A time scale $\mathbb{T}$ is called an almost periodic time scale if

$$
\Pi:=\{\tau \in \mathbb{R}: t \pm \tau \in \mathbb{T}, \forall t \in \mathbb{T}\} \neq\{0\} .
$$

Remark 2. The concept of almost periodic time scales proposed in [1] was cited by [7] to introduce the definition of almost automorphic functions on time scales which can be applied to study almost automorphic solutions of dynamic equations on time scales, and $\mathbb{T}$ is also called invariant time scale under translations in Definition 3.1 of [7]. In fact, we find that Definition 1 is equivalent to Definition 1.1 proposed in [15]; that is, the almost periodic time scale $\mathbb{T}$ proposed in [1] is a periodic time scale. In this paper, we will give three more general and accurate equivalent concepts of almost periodic time scales and redefine the concepts of almost periodic functions on this new time scale concept. Furthermore, some 
examples and applications will be shown in which our results can be applied to iron out the flaws of the proposed definition in [1].

We give some notations; $\mathbb{E}^{n}$ denote $\mathbb{R}^{n}$ or $\mathbb{C}^{n}, D$ denotes an open set in $\mathbb{E}^{n}$ or $D=\mathbb{E}^{n}$, and $S$ denotes an arbitrary compact subset of $D$.

Definition 3 (see [1]). Let $\mathbb{T}$ be an almost periodic time scale. A function $f \in C\left(\mathbb{T} \times D, \mathbb{E}^{n}\right)$ is called an almost periodic function in $t \in \mathbb{T}$ uniformly for $x \in D$ if the $\varepsilon$-translation set of $f$

$$
\begin{gathered}
E\{\varepsilon, f, S\}=\{\tau \in \Pi:|f(t+\tau, x)-f(t, x)|<\varepsilon, \\
\forall(t, x) \in \mathbb{T} \times S\}
\end{gathered}
$$

is relatively dense set in $\mathbb{T}$ for all $\varepsilon>0$ and for each compact subset $S$ of $D$; that is, for any given $\varepsilon>0$ and each compact subset $S$ of $D$, there exists a constant $l(\varepsilon, S)>0$ such that each interval of length $l(\varepsilon, S)$ contains a $\tau(\varepsilon, S) \in E\{\varepsilon, f, S\}$ such that

$$
|f(t+\tau, x)-f(t, x)|<\varepsilon, \quad \forall(t, x) \in \mathbb{T} \times S .
$$

$\tau$ is called the $\varepsilon$-translation number of $f$ and and $l(\varepsilon)$ is called the inclusion length of $E\{\varepsilon, f, S\}$.

For convenience, we denote $A P(\mathbb{T})=\left\{f \in C\left(\mathbb{T}, \mathbb{E}^{n}\right)\right.$ : $f$ is almost periodic $\}$ and introduce some notations: let $\alpha=$ $\left\{\alpha_{n}\right\}$ and $\beta=\left\{\beta_{n}\right\}$ be two sequences. Then $\beta \subset \alpha$ means that $\beta$ is a subsequence of $\alpha$; $\alpha+\beta=\left\{\alpha_{n}+\beta_{n}\right\},-\alpha=\left\{-\alpha_{n}\right\}$, and $\alpha$ and $\beta$ are common subsequences of $\alpha^{\prime}$ and $\beta^{\prime}$, respectively, means that $\alpha_{n}=\alpha_{n(k)}^{\prime}$ and $\beta_{n}=\beta_{n(k)}^{\prime}$ for some given function $n(k)$.

We will introduce the translation operator $T$; $T_{\alpha} f(t, x)=$ $g(t, x)$ means that $g(t, x)=\lim _{n \rightarrow+\infty} f\left(t+\alpha_{n}, x\right)$ and is written only when the limit exists.

Definition 4 (see [1]). Let $f(t, x) \in C\left(\mathbb{T} \times D, \mathbb{E}^{n}\right)$, if, for any given sequence $\alpha^{\prime} \subset \Pi$, there exists a subsequence $\alpha \subset \alpha^{\prime}$ such that $T_{\alpha} f(t, x)$ exists uniformly on $\mathbb{T} \times S$; then, $f(t, x)$ is called an almost periodic function in $t$ uniformly for $x \in D$.

However, in [15], the authors propose the definition of periodic time scales and give the remark as follows.

Definition 5 (see [15]). One can say that a time scale $\mathbb{T}$ is periodic if there exists $p>0$ such that if $t \in \mathbb{T}$ then $t \pm p \in \mathbb{T}$. For $\mathbb{T} \neq \mathbb{R}$, the smallest positive $p$ is called the period of the time scale.

Remark 6 (see [15]). If $\mathbb{T}$ is a periodic time scale with period $p$, then $\sigma(t+n p)=\sigma(t)+n p$. Consequently, the graininess function $\mu$ satisfies $\mu(t+n p)=\sigma(t+n p)-(t+n p)=\sigma(t)-t=$ $\mu(t)$ and so it is a periodic function with period $p$.

Note that Definitions 3 and 4 are proposed based on the set $\Pi$. Although Definition 1 is exactly like Definition 5 since $\forall \tau \in \Pi$, one has $t \pm \tau \in \mathbb{T}$. In order to clarify some theoretical ambiguities between periodic time scales in [15] and almost periodic time scales in [1], in the following, we will propose a more general and accurate concept of almost periodic time scales instead of Definition 1 and give some examples of time scales which are almost periodic but not periodic.

Let $\tau$ be a number. We set the time scales as follows:

$$
\begin{gathered}
\mathbb{T}:=\bigcup_{i=-\infty}^{+\infty}\left[\alpha_{i}, \beta_{i}\right], \\
\mathbb{T}^{\tau}:=\mathbb{T}+\tau=\{t+\tau: \forall t \in \mathbb{T}\}:=\bigcup_{i=-\infty}^{+\infty}\left[\alpha_{i}^{\tau}, \beta_{i}^{\tau}\right] .
\end{gathered}
$$

Define the distance between two time scales, $\mathbb{T}$ and $\mathbb{T}^{\tau}$, by

$$
d\left(\mathbb{T}, \mathbb{T}^{\tau}\right)=\max \left\{\sup _{i \in \mathbb{Z}}\left|\alpha_{i}-\alpha_{i}^{\tau}\right|, \sup _{i \in \mathbb{Z}}\left|\beta_{i}-\beta_{i}^{\tau}\right|\right\} .
$$

Definition 7 (see [19]). A subset $S$ of $\mathbb{R}$ is called relatively dense if there exists a positive number $L$ such that $[a, a+L] \cap$ $S \neq \emptyset$ for all $a \in \mathbb{R}$. The number $L$ is called the inclusion length.

Definition 8. We say $\mathbb{T}$ is an almost periodic time scale if, for any give $\varepsilon>0$, there exists a constant $l(\varepsilon)>0$ such that each interval of length $l(\varepsilon)$ contains $a \tau(\varepsilon)$ such that

$$
d\left(\mathbb{T}, \mathbb{T}^{\tau}\right)<\varepsilon
$$

that is, for any $\varepsilon>0$, the following set

$$
E\{\mathbb{T}, \varepsilon\}=\left\{\tau \in \mathbb{R}: d\left(\mathbb{T}^{\tau}, \mathbb{T}\right)<\varepsilon\right\}
$$

is relatively dense. $\tau$ is called the $\varepsilon$-translation number of $\mathbb{T}$ and $l(\varepsilon)$ is called the inclusion length of $E\{\mathbb{T}, \varepsilon\}$, and $E\{\mathbb{T}, \varepsilon\}$ is called the $\varepsilon$-translation set of $\mathbb{T}$.

Remark 9. From Definition 1 , one can easily see that if $\Pi \neq \emptyset$, then for any $\varepsilon>0$, there exists a constant $l(\varepsilon)>0$ such that each interval of length $l(\varepsilon)$ contains a $\tau(\varepsilon) \in \Pi$ such that

$$
\mathbb{T} \cap \mathbb{T}^{\tau}=\mathbb{T}, \quad d\left(\mathbb{T}, \mathbb{T}^{\tau}\right)=0<\varepsilon .
$$

Therefore, Definition 8 includes Definition 1. Particularly, it is worth emphasising that $\tau(\varepsilon)$ in Definition 8 need not satisfy $t \pm \tau(\varepsilon) \in \mathbb{T}$ for all $t \in \mathbb{T}$.

Remark 10. According to Definition 8, one can obtain that $\sup \mathbb{T}=+\infty$, inf $\mathbb{T}=-\infty$, and

$$
\begin{aligned}
& \mathbb{T} \cap \mathbb{\mathbb { V }}^{\tau} \neq \emptyset, \quad \sup \left\{\mathbb{T} \cap \mathbb{\mathbb { T }}^{\tau}\right\}=+\infty, \\
& \inf \left\{\mathbb{T} \cap \mathbb{T}^{\tau}\right\}=-\infty \text {. }
\end{aligned}
$$

Furthermore, in Definition 8 , one can see that if $d\left(\mathbb{T}, \mathbb{T}^{\tau}\right)<\varepsilon$, then $d\left(\mathbb{T}, \mathbb{T}^{-\tau}\right)<\varepsilon$; that is, if $\tau \in E\{\mathbb{T}, \varepsilon\}$, then $-\tau \in E\{\mathbb{T}, \varepsilon\}$. If $\tau_{1} \in E\{\mathbb{T}, \varepsilon\}, \tau_{2} \in E\{\mathbb{T}, \varepsilon\}$, then we have $\tau_{1}+\tau_{2} \in E\{\mathbb{T}, 2 \varepsilon\}$ since

$$
\begin{aligned}
d\left(\mathbb{T}, \mathbb{T}^{\tau_{1}+\tau_{2}}\right) & \leq d\left(\mathbb{T}, \mathbb{T}^{\tau_{1}}\right)+d\left(\mathbb{T}^{\tau_{1}}, \mathbb{T}^{\tau_{1}+\tau_{2}}\right) \\
& =d\left(\mathbb{T}, \mathbb{T}^{\tau_{1}}\right)+d\left(\mathbb{T}, \mathbb{T}^{\tau_{2}}\right)<2 \varepsilon .
\end{aligned}
$$


Theorem 11. Let $\mathbb{T}$ be an almost periodic time scale. Then for any given sequence $\alpha^{\prime}$, there exists a subsequence $\alpha \subset \alpha^{\prime}$ such that $\left\{\mathbb{T}^{\alpha^{n}}\right\}$ converges to a time scale $\mathbb{T}_{0}$; that is, for any given $\varepsilon>$ 0 , there exists $N_{0}>0$ such that $n>N_{0}$ implies $d\left(\mathbb{T}^{\alpha_{n}}, \mathbb{T}_{0}\right)<\varepsilon$. Furthermore, $\mathbb{T}_{0}$ is also almost periodic.

Proof. For any $\varepsilon>0$. Let $l=l(\varepsilon / 4)$ be an inclusion length of $E\{\mathbb{T}, \varepsilon / 4\}$. For any given subsequence $\alpha^{\prime}=\left\{\alpha_{n}^{\prime}\right\}$, we denote $\alpha_{n}^{\prime}=\tau_{n}^{\prime}+\gamma_{n}^{\prime}$, where $\tau_{n}^{\prime} \in E\{\mathbb{T}, \varepsilon / 4\}$ and $0 \leq \gamma_{n}^{\prime} \leq l, n=1,2, \ldots$. Therefore, there exists a subsequence $\gamma=\left\{\gamma_{n}\right\} \subset \gamma^{\prime}=\left\{\gamma_{n}^{\prime}\right\}$ such that $\gamma_{n} \rightarrow s$ as $n \rightarrow \infty, 0 \leq s \leq l$.

Also, it is easy to see that there exists $\delta(\varepsilon)>0$ so that $\left|t_{1}-t_{2}\right|<\delta$ implies

$$
d\left(\mathbb{T}^{t_{1}}, \mathbb{T}^{t_{2}}\right)<\frac{\varepsilon}{2}
$$

Since $\gamma$ is a convergent sequence, there exists $N=N(\delta)$ so that $p, m \geq N$ implies $\left|\gamma_{p}-\gamma_{m}\right|<\delta$. Now, one can take $\alpha \subset \alpha^{\prime}, \tau \subset \tau^{\prime}=\left\{\tau_{n}^{\prime}\right\}$ such that $\alpha, \tau$ are common with $\gamma$, and then for any integers $p, m \geq N$, we have

$$
\begin{aligned}
d\left(\mathbb{T}^{\tau_{p}-\tau_{m}}, \mathbb{T}\right) & \leq d\left(\mathbb{T}^{\tau_{p}-\tau_{m}}, \mathbb{T}^{\tau_{p}}\right)+d\left(\mathbb{T}^{\tau_{p}}, \mathbb{T}\right) \\
& <\frac{\varepsilon}{4}+\frac{\varepsilon}{4}=\frac{\varepsilon}{2}
\end{aligned}
$$

that is,

$$
\left(\alpha_{p}-\alpha_{m}\right)-\left(\gamma_{p}-\gamma_{m}\right)=\tau_{p}-\tau_{m} \in E\left\{\mathbb{T}, \frac{\varepsilon}{2}\right\} .
$$

Hence, we can obtain

$$
\begin{aligned}
d\left(\mathbb{T}^{\alpha_{p}}, \mathbb{T}^{\alpha_{m}}\right) & \leq d\left(\mathbb{T}^{\alpha_{p}-\alpha_{m}}, \mathbb{T}\right) \\
& \leq d\left(\mathbb{T}^{\alpha_{p}-\alpha_{m}}, \mathbb{T}^{\gamma_{p}-\gamma_{m}}\right)+d\left(\mathbb{T}^{\gamma_{p}-\gamma_{m}}, \mathbb{T}\right) \\
& <\frac{\varepsilon}{2}+\frac{\varepsilon}{2}=\varepsilon .
\end{aligned}
$$

Thus, we can take sequences $\alpha^{(k)}=\left\{\alpha_{n}^{(k)}\right\}, k=1,2, \ldots$, and $\alpha^{(k+1)} \subset \alpha^{(k)} \subset \alpha$ such that for any integers $m, p$ the following holds:

$$
d\left(\mathbb{T}^{\alpha_{p}^{(k)}}, \mathbb{T}^{\alpha_{m}^{(k)}}\right)<\frac{1}{k}, \quad k=1,2, \ldots
$$

For all sequences $\alpha^{(k)}, k=1,2, \ldots$, we can take a sequence $\beta=\left\{\beta_{n}\right\}, \beta_{n}=\alpha_{n}^{(n)}$, and then it is easy to see that $\left\{\mathbb{T}^{\beta_{n}}\right\} \subset\left\{\mathbb{T}^{\alpha_{n}}\right\}$ for any integers $p, m$ with $p<m$ the following holds:

$$
d\left(\mathbb{T}^{\beta_{p}}, \mathbb{T}^{\beta_{m}}\right)<\frac{1}{p} .
$$

Therefore, $\left\{\mathbb{T}^{\beta_{n}}\right\}$ converges to some $\mathbb{T}_{0}$ which is a closed subset of $\mathbb{R}$; that is, $d\left(\mathbb{T}^{\beta_{n}}, \mathbb{T}_{0}\right) \rightarrow 0$ as $n \rightarrow \infty$.

Finally, for any given $\varepsilon>0$, one can take $\tau \in E\{\mathbb{T}, \varepsilon\}$; then, the following holds:

$$
d\left(\mathbb{T}^{\beta_{n}+\tau}, \mathbb{T}^{\beta_{n}}\right)<\varepsilon
$$

Letting $n \rightarrow+\infty$, we have

$$
d\left(\mathbb{T}_{0}^{\tau}, \mathbb{T}_{0}\right) \leq \varepsilon
$$

which implies that $E\left\{\mathbb{T}_{0}, \varepsilon\right\}$ is relatively dense. Therefore, $\mathbb{T}_{0}$ is almost periodic. This completes the proof.

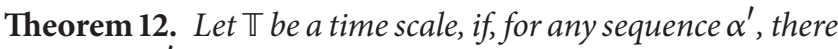
exists $\alpha \subset \alpha^{\prime}$ such that $\left\{\mathbb{T}^{\alpha_{n}}\right\}$ converges to a time scale $\mathbb{T}_{0}$, then $\mathbb{}$ is almost periodic.

Proof. For contradiction, if this is not true, then there exists $\varepsilon_{0}>0$ such that for any sufficiently large $l>0$, we can find an interval with length of $l$ and there is no $\varepsilon_{0}$-translation numbers of $\mathbb{T}$ in this interval; that is, every point in this interval is not in $E\left\{\mathbb{T}, \varepsilon_{0}\right\}$.

One can take a number $\alpha_{1}^{\prime}$ and find an interval $\left(a_{1}, b_{1}\right)$ with $b_{1}-a_{1}>2\left|\alpha_{1}^{\prime}\right|$, where $a_{1}, b_{1}$ satisfy that there is no $\varepsilon_{0}$ translation numbers of $\mathbb{T}$ in the interval $\left(a_{1}, b_{1}\right)$. Next, taking $\alpha_{2}^{\prime}=(1 / 2)\left(a_{1}+b_{1}\right)$, obviously, $\alpha_{2}^{\prime}-\alpha_{1}^{\prime} \in\left(a_{1}, b_{1}\right)$, so $\alpha_{2}^{\prime}-$ $\alpha_{1}^{\prime} \notin E\left\{\mathbb{T}, \varepsilon_{0}\right\}$; then, one can find an interval $\left(a_{2}, b_{2}\right)$ with $b_{2}-$ $a_{2}>2\left(\left|\alpha_{1}^{\prime}\right|+\left|\alpha_{2}^{\prime}\right|\right)$, where $a_{2}, b_{2}$ satisfy that there is no $\varepsilon_{0^{-}}$ translation numbers of $\mathbb{T}$ in the interval $\left(a_{2}, b_{2}\right)$. Next, taking $\alpha_{3}^{\prime}=(1 / 2)\left(a_{2}+b_{2}\right)$, obviously, $\alpha_{3}^{\prime}-\alpha_{2}^{\prime}, \alpha_{3}^{\prime}-\alpha_{1}^{\prime} \notin E\left\{\mathbb{T}, \varepsilon_{0}\right\}$. One can repeat these processes again and again and find $\alpha_{4}^{\prime}, \alpha_{5}^{\prime}, \ldots$, such that $\alpha_{i}^{\prime}-\alpha_{j}^{\prime} \notin E\left\{\mathbb{T}, \varepsilon_{0}\right\}, i>j$. Hence, for any $i \neq j, i, j=$ $1,2, \ldots$, without loss of generality, letting $i>j$, we have

$$
d\left(\mathbb{T}^{\alpha_{i}^{\prime}}, \mathbb{T}^{\alpha_{j}^{\prime}}\right)=d\left(\mathbb{T}^{\alpha_{i}^{\prime}-\alpha_{j}^{\prime}}, \mathbb{T}\right) \geq \varepsilon_{0} .
$$

Therefore, there is no convergent subsequence of $\left\{\mathbb{T}^{\alpha_{n}^{\prime}}\right\}$, a contradiction. Hence, $\mathbb{T}$ is almost periodic. This completes the proof.

From Theorems 11 and 12, we can obtain the following equivalent definition of almost periodic time scales.

Definition 13. Let $\mathbb{T}$ be a time scale, and if, for any given sequence $\alpha^{\prime}$, there exists a subsequence $\alpha \subset \alpha^{\prime}$ such that $\left\{\mathbb{T}^{\alpha_{n}}\right\}$ converges to a time scale $\mathbb{T}_{0}$, then $\mathbb{T}$ is called an almost periodic time scale.

In the sequel, based on Definitions 8 and 13, we will give the two equivalent concepts of almost periodic functions on time scales.

Definition 14. Let $\mathbb{T}$ be an almost periodic time scale. A function $f \in C\left(\mathbb{T} \times D, \mathbb{E}^{n}\right)$ is called an almost periodic function in $t \in \mathbb{T}$ uniformly for $x \in D$ if the $\varepsilon$-translation set of $f$

$$
\begin{gathered}
E\{\varepsilon, f, S\}=\{\tau \in \mathbb{R}:|f(t+\tau, x)-f(t, x)|<\varepsilon, \\
\left.\forall(t, x) \in\left(\mathbb{T} \cap \mathbb{T}^{-\tau}\right) \times S\right\}
\end{gathered}
$$

is a relatively dense set for all $\varepsilon>0$ and for each compact subset $S$ of $D$; that is, for any given $\varepsilon>0$ and each compact subset $S$ of $D$, there exists a constant $l(\varepsilon, S)>0$ such that each interval of length $l(\varepsilon, S)$ contains a $\tau(\varepsilon, S) \in E\{\varepsilon, f, S\}$ such that

$$
|f(t+\tau, x)-f(t, x)|<\varepsilon, \quad \forall(t, x) \in\left(\mathbb{T} \cap \mathbb{\mathbb { T }}^{-\tau}\right) \times S .
$$

$\tau$ is called the $\varepsilon$-translation number of $f$ and $l(\varepsilon)$ is called the inclusion length of $E\{\varepsilon, f, S\}$. 
Remark 15. From Definition 14, one can easily see that if $\mathbb{T}$ is a periodic time scale, that is, $\mathbb{T}$ satisfies Definition 1 , then in Definition 14, we have $\mathbb{T} \cap \mathbb{T}^{-\tau}=\mathbb{T}$. Hence, Definition 14 strictly includes Definition 3.

Definition 16. Assume that $\mathbb{T}$ is an almost periodic time scale. Let $f(t, x) \in C\left(\mathbb{T} \times D, \mathbb{E}^{n}\right)$, if for any given sequence $\alpha^{\prime}$, there exists a subsequence $\alpha \subset \alpha^{\prime}$ such that the limit set $\mathbb{T}_{0}$ of $\left\{\mathbb{T}^{-\alpha n}\right\}$ exists and $\mathbb{T}_{\alpha} f(t, x)$ exists uniformly on $\mathbb{T}_{0} \times S$, then $f(t, x)$ is called an almost periodic function in $t$ uniformly for $x \in D$.

Remark 17. Noting that $\mathbb{T}$ is almost periodic, according to Definition 13, we have

$$
\lim _{n \rightarrow \infty} \mathbb{T}^{-\alpha_{n}}=\bigcap_{j=1}^{\infty} \bigcup_{k=j}^{\infty} \mathbb{T}^{-\alpha_{n}}=\bigcup_{j=1}^{\infty} \bigcap_{k=j}^{\infty} \mathbb{T}^{-\alpha_{n}}=\mathbb{T}_{0} .
$$

Therefore, we can also substitute $\bigcap_{j=1}^{\infty} \bigcup_{k=j}^{\infty} \mathbb{T}^{-\alpha_{n}}$ or $\bigcup_{j=1}^{\infty} \bigcap_{k=j}^{\infty} \mathbb{T}^{-\alpha_{n}}$ for $\mathbb{T}_{0}$ in Definition 16. Furthermore, one can see that if $\mathbb{T}$ is periodic, then we have $\mathbb{T}_{0}=\bigcap_{j=1}^{\infty} \bigcup_{k=j}^{\infty} \mathbb{T}^{-\alpha_{n}}=\bigcup_{j=1}^{\infty} \bigcap_{k=j}^{\infty} \mathbb{T}^{-\alpha_{n}}=\mathbb{T}$, that is, Definition 16 strictly includes Definition 4.

From Definition 8 and the definition of the graininess function $\mu$, one can have the following.

Theorem 18. If $\mathbb{T}$ is an almost periodic time scale, then for any $\varepsilon>0$ there exists a constant $l(\varepsilon)>0$ such that each interval of length $l(\varepsilon)$ contains a $\tau(\varepsilon) \in E\{\varepsilon, \mu\}$ such that

$$
|\mu(t+\tau)-\mu(t)|<\varepsilon, \quad \forall t \in \mathbb{T} \cap \mathbb{T}^{-\tau} .
$$

Remark 19. The inequality (31) can also be written as

$$
|\sigma(t+\tau)-\sigma(t)-\tau|<\varepsilon, \quad \forall t \in \mathbb{T} \cap \mathbb{T}^{-\tau},
$$

which indicates that if $\mathbb{T}$ is $\tau$-periodic, we have $\sigma(t+\tau)=$ $\sigma(t)+\tau$; then, $\mathbb{T}$ is an almost periodic time scale.

Remark 20. Conversely, if the graininess function $\mu$ is an almost periodic function, from the definition of the function $\mu: \mathbb{T} \rightarrow \mathbb{R}$, that is, $\mu(t)=\sigma(t)-t$, one can obviously see that there must exist at least $a \tau \in E\{\varepsilon, \mu\}$ in the each interval of length $l(\varepsilon)$ such that $d\left(\mathbb{T}, \mathbb{T}^{-\tau}\right)<\varepsilon$. Therefore, we can easily get that $\mathbb{T}$ is an almost periodic time scale by Definition 8 . According to this, in the following, we will introduce the third definition of almost periodic time scales which is equivalent to Definition 8.

Now, we give the third concept of almost periodic time scales by the graininess function $\mu$ as follows.

Definition 21. Let $\mu: \mathbb{T} \rightarrow \mathbb{R}, \mu(t)=\sigma(t)-t$. One can say that $\mathbb{Z}$ is an almost periodic time scale if, for any $\varepsilon>0$, the set

$$
\Pi^{*}=\left\{\tau \in \mathbb{R}:|\mu(t+\tau)-\mu(t)|<\varepsilon, \forall t \in \mathbb{T} \cap \mathbb{T}^{-\tau}\right\}
$$

is relatively dense; that is, $\mu$ is an almost periodic function on $\mathbb{T}$.

By Theorem 18 and Definition 21, we can get the following corollaries.
Corollary 22. If $\mathbb{T} \neq \mathbb{R}$ and $\mathbb{T}$ is a periodic time scale, then $\mathbb{T}$ has the smallest positive period $p$ and the graininess function $\mu$ is a periodic function with period $p$.

Corollary 23. All periodic time scales are almost periodic.

Corollary 24. $\mathbb{T}$ is an $\tau$-periodic time scale if and only if the graininess function $\mu: \mathbb{T} \rightarrow \mathbb{R}^{+}$is a $\tau$-periodic function.

Next, we will show some examples of almost periodic time scales.

Example 25. If $\mathbb{T}=\bigcup_{k \in \mathbb{Z}}[k(a+b), k(a+b)+b]$, where $a \neq-b$, then

$$
\begin{gathered}
\sigma(t)= \begin{cases}t, & \text { if } t \in \bigcup_{k=0}^{\infty}[k(a+b), k(a+b)+b), \\
t+a, & \text { if } t \in \bigcup_{k=0}^{\infty}\{k(a+b)+b\},\end{cases} \\
\mu(t)= \begin{cases}0, & \text { if } t \in \bigcup_{k=0}^{\infty}[k(a+b), k(a+b)+b), \\
a, & \text { if } t \in \bigcup_{k=0}^{\infty}\{k(a+b)+b\} .\end{cases}
\end{gathered}
$$

Thus, $\mathbb{T}$ is almost periodic. Obviously, if $b=0, a=1$, then $\mathbb{T}=\mathbb{Z}$. if $b=1, a=0$, then $\mathbb{T}=\mathbb{R}$.

Remark 26. One can easily see that Example 25 is a periodic time scale with periodicity $a+b$, and by Corollary 23 , it is also an almost periodic time scale.

(1) As everyone knows, the graininess function $\mu: \mathbb{T} \rightarrow$ $[0, \infty)$ defined by

$$
\mu(t):=\sigma(t)-t
$$

can describe the construction of a time scale. From Definition 21, one can see that $\mu(t)$ is an almost periodic function if and only if $\mathbb{T}$ is an almost periodic time scale; on the other hand, from Definition 5 and Corollary $24, \mathbb{T}$ is a periodic time scale if and only if $\mu: \mathbb{T} \rightarrow \mathbb{R}^{+}$is a periodic function. Hence, from the graininess function $\mu$, we can see that these two time scales are different and we will show some examples in the next point.

(2) In this point, we will show some examples of time scales which are almost periodic but not periodic.

Example 27. Let $a>1$ and consider the the following time scale:

$$
\mathbb{P}_{a, \cos t}=\bigcup_{m=1}^{\infty}\left[p_{m}, a+p_{m}\right]
$$

where 


$$
p_{m}=(m-1) a+\sum_{k=1}^{m-1} \cos (\underbrace{k a+\cos a+\cos (2 a+\cos a)+\cdots+\cos ((k-1) a+\cos a)}_{k \text { terms }}) .
$$

Then, we have

$$
\begin{gathered}
\sigma(t)= \begin{cases}t, & \text { if } t \in \bigcup_{m=1}^{\infty}\left[p_{m}, a+p_{m}\right), \\
t+\cos t, & \text { if } t \in \bigcup_{m=1}^{\infty}\left\{a+p_{m}\right\},\end{cases} \\
\mu(t)= \begin{cases}0, & \text { if } t \in \bigcup_{m=1}^{\infty}\left[p_{m}, a+p_{m}\right), \\
\cos t, & \text { if } t \in \bigcup_{m=1}^{\infty}\left\{a+p_{m}\right\} .\end{cases}
\end{gathered}
$$

One can see that this kind of time scale has the graininess function $\mu$ which is an almost periodic function, and by Definition $21, \mathbb{T}$ is an almost periodic time scale. It is worth noting that there is not any $\tau \in \mathbb{R}$ such that $t \pm \tau \in \mathbb{T}$ for all $t \in \mathbb{T}$; thus, $\mathbb{T}$ is not a periodic time scale by Definitions 1 or 5 .

Example 28. Let $a>1$ and consider the the following time scale:

$$
\mathbb{P}_{a, \cos t+\cos \sqrt{2} t}=\bigcup_{m=1}^{\infty}\left[p_{m}, a+p_{m}\right]
$$

where

$$
\begin{aligned}
& p_{m}=(m-1) a \\
&+\sum_{k=1}^{m-1} \cos (k a+\cos a+\cos \sqrt{2} a \\
& \\
&+\cos (2 a+\cos a+\cos \sqrt{2} a)
\end{aligned}
$$

$$
+\cdots+\cos ((k-1) a+\cos a+\cos \sqrt{2} a)
$$$$
\underbrace{+\cos \sqrt{2((k-1) a+\cos a+\cos \sqrt{2} a)}}_{2 k+1 \text { terms }}) \text {. }
$$

Then, we have

$$
\begin{gathered}
\sigma(t)= \begin{cases}t, & \text { if } t \in \bigcup_{m=1}^{\infty}\left[p_{m}, a+p_{m}\right), \\
t+\cos t+\cos \sqrt{2} t, & \text { if } t \in \bigcup_{m=1}^{\infty}\left\{a+p_{m}\right\},\end{cases} \\
\mu(t)= \begin{cases}0, & \text { if } t \in \bigcup_{m=1}^{\infty}\left[p_{m}, a+p_{m}\right), \\
\cos t+\cos \sqrt{2} t, & \text { if } t \in \bigcup_{m=1}^{\infty}\left\{a+p_{m}\right\} .\end{cases}
\end{gathered}
$$

We see that this kind of time scale has the graininess function $\mu$ which is an almost periodic function, and by Definition 21 , $\mathbb{T}$ is an almost periodic time scale. It is worth noting that there is not any $\tau \in \mathbb{R}$ such that $t \pm \tau \in \mathbb{T}$ for all $t \in \mathbb{T}$; thus, $\mathbb{T}$ is not a periodic time scale by Definitions 1 or 5 .

Example 29. Let $a>1$ and consider the the following time scale:

$$
\mathbb{P}_{a, \sin t}=\bigcup_{m=1}^{\infty}\left[p_{m}, a+p_{m}\right]
$$

where

$$
p_{m}=(m-1) a+\sum_{k=1}^{m-1} \sin (\underbrace{k a+\sin a+\sin (2 a+\sin a)+\cdots+\sin ((k-1) a+\sin a)}_{k \text { terms }}) .
$$

Then, we have

$$
\begin{gathered}
\sigma(t)= \begin{cases}t, & \text { if } t \in \bigcup_{m=1}^{\infty}\left[p_{m}, a+p_{m}\right), \\
t+\sin t, & \text { if } t \in \bigcup_{m=1}^{\infty}\left\{a+p_{m}\right\},\end{cases} \\
\mu(t)= \begin{cases}0, & \text { if } t \in \bigcup_{m=1}^{\infty}\left[p_{m}, a+p_{m}\right), \\
\sin t, & \text { if } t \in \bigcup_{m=1}^{\infty}\left\{a+p_{m}\right\} .\end{cases}
\end{gathered}
$$

One can see that this kind of time scale has the graininess function $\mu$ which is an almost periodic function, and by Definition 21, $\mathbb{T}$ is an almost periodic time scale. It is worth noting that there is not any $\tau \in \mathbb{R}$ such that $t \pm \tau \in \mathbb{Z}$ for all $t \in \mathbb{T}$; thus, $\mathbb{T}$ is not a periodic time scale by Definitions 1 or 5 .

Example 30. Let $a>1$ and consider the the following time scale:

$$
\mathbb{P}_{a, \sin t+\sin \sqrt{2} t}=\bigcup_{m=1}^{\infty}\left[p_{m}, a+p_{m}\right]
$$


where

$$
\begin{aligned}
& p_{m}=(m-1) a \\
&+\sum_{k=1}^{m-1} \sin (k a+\sin a+\sin \sqrt{2} a \\
&+\sin (2 a+\sin a+\sin \sqrt{2} a) \\
&+\ldots+\sin ((k-1) a+\sin a+\sin \sqrt{2} a) \\
&\quad \underbrace{+\sin \sqrt{2}((k-1) a+\sin a+\sin \sqrt{2} a)}_{2 k+1 \text { terms }}) .
\end{aligned}
$$

Then, we have

$$
\begin{gathered}
\sigma(t)= \begin{cases}t, & \text { if } t \in \bigcup_{m=1}^{\infty}\left[p_{m}, a+p_{m}\right), \\
t+\sin t+\sin \sqrt{2} t, & \text { if } t \in \bigcup_{m=1}^{\infty}\left\{a+p_{m}\right\},\end{cases} \\
\mu(t)= \begin{cases}0, & \text { if } t \in \bigcup_{m=1}^{\infty}\left[p_{m}, a+p_{m}\right), \\
\sin t+\sin \sqrt{2} t, & \text { if } t \in \bigcup_{m=1}^{\infty}\left\{a+p_{m}\right\} .\end{cases}
\end{gathered}
$$

We see that this kind of time scale has the graininess function $\mu$ which is an almost periodic function, and by Definition 21, $\mathbb{T}$ is an almost periodic time scale. It is worth noting that there is not any $\tau \in \mathbb{R}$ such that $t \pm \tau \in \mathbb{T}$ for all $t \in \mathbb{T}$; thus, $\mathbb{T}$ is not a periodic time scale by Definition 1 or Definition 5 .

Remark 31. From Example 27 to Example 30, there is not any $\tau \in \mathbb{R}$ such that $t \pm \tau \in \mathbb{T}$ for all $t \in \mathbb{T}$. Therefore, all examples show that the concepts of almost periodic time scales proposed in this paper strictly include all periodic time scales and they are more general and accurate.

By Definitions 8 and 21, we can give the following sufficient and necessary condition to guarantee that $\mathbb{T}$ is almost periodic.

Theorem 32. Let $\mathbb{T}$ be a time scale and $\mathbb{T}$ is almost periodic if and only if $\Pi^{*}$ is relatively dense in $\mathbb{T}$; that is, $\mu$ is an almost periodic function on $\mathbb{T}$.

Corollary 33. The time scales are invariant under translations if and only if $\mu$ is periodic.

Remark 34. The Examples 3.7, 3.8, 3.9, 3.10, and 3.11 in [7] are invariant time scales under translations, and obviously, all of them are periodic time scales.

\section{Cauchy Matrix for Dynamic Equations}

In this section, by the new concepts proposed in Section 2, we will prove some useful theorems and lemmas of Cauchy matrix of (1), and using these results, we can obtain the existence and uniqueness of almost periodic solutions of (2) straightly. These theorems and lemmas can be applied to study almost periodic solutions of many other types of mathematical models on time scales.

Theorem 35. Let $W(t)$ be a fundamental matrix of system (1). Then, for $t \geq t_{0}$, every solution of system (2) is given by

$$
x(t)=W(t)\left[c+\int_{t_{0}}^{t} W^{-1}(\sigma(\tau)) f(\tau, x(\tau)) \Delta \tau\right] .
$$

In particular, if $W(t)=W\left(t, t_{0}\right)$ is a Cauchy matrix of system (1), then, for $t \geq t_{0}$, any solution of (2) with initial condition $x\left(t_{0}, x_{0}\right)=x_{0}$ can be written as

$$
x\left(t, x_{0}\right)=W\left(t, t_{0}\right) x_{0}+\int_{t_{0}}^{t} W(t, \sigma(\tau)) f(\tau, x(\tau)) \Delta \tau .
$$

Proof. Since $W$ is a nonsingular matrix and $\Delta$-differentiable. Then, under the linear change of variables,

$$
x=W(t) y ;
$$

then, system (2) turns into the following:

$$
y^{\Delta}=W^{-1}(\sigma(t)) f(t, x(t)) .
$$

From (51) we can find that, for $t \geq t_{0}$,

$$
y(t)=c+\int_{t_{0}}^{t} W^{-1}(\sigma(\tau)) f(\tau, x(\tau)) \Delta \tau
$$

where $c=y\left(t_{0}\right)$ is a constant vector; that is,

$$
W^{-1}(t) x=W^{-1}\left(t_{0}\right) x_{0}+\int_{t_{0}}^{t} W^{-1}(\sigma(\tau)) f(\tau, x(\tau)) \Delta \tau ;
$$

then, we can get (48) and (49). This completes the proof.

Remark 36. By Theorem 35, one can easily check that (2) has a bounded solution as follows:

$$
x(t)=\int_{-\infty}^{t} W(t, \sigma(s)) f(s, x(s)) \Delta s .
$$

Noting that if (1) admits an exponential dichotomy, then we can take the projection $P=I$ in Lemma 2.13 of [2] and $W(t, \sigma(s))=X(t) X^{-1}(\sigma(s))$ to get this bounded solution. Furthermore, by the concept of exponential dichotomies on time scales, one can easily see that there exist $K>1, \alpha>0$ such that

$$
\|W(t, \sigma(s))\|=\left\|X(t) X^{-1}(\sigma(s))\right\| \leq K e_{\ominus \alpha}(t, s), \quad t \geq s,
$$

where $X(t)$ is the fundamental solution matrix of (1); that is, (5) holds. 
Definition 37 (see [16]). Let $f: \mathbb{T} \rightarrow \mathbb{R}$ be a function and let $t=\left(t_{1}, t_{2}, \ldots, t_{n}\right) \in \mathbb{T}^{\kappa}$. Then define $f^{\Delta_{i}}(t)$ to be the number (provided it exists) with the property that given any $\varepsilon>0$ there exists a neighborhood $U$ of $t_{i}$ with $U=\left(t_{i}-\delta, t_{i}+\delta\right) \cap \mathbb{T}_{i}$ for $\delta>0$ such that

$$
\begin{aligned}
& \left|\left[f^{\sigma_{i}}(t)-f_{i}^{s}(t)\right]-f^{\Delta_{i}}(t)\left[\sigma_{i}(t)-s\right]\right| \\
& \quad \leq \varepsilon\left|\sigma_{i}(t)-s\right| \quad \forall s \in U .
\end{aligned}
$$

$f^{\Delta_{i}}$ is called the partial delta derivative of $f$ at $t$ with respect to the variable $t_{i}$.

Theorem 38. For system (1), let the matrix $A \in C\left(\mathbb{T}, \mathbb{R}^{n \times n}\right)$ be almost periodic. If the Cauchy matrix $W(t, s)$ satisfies the inequality

$$
\|W(t, s)\| \leq K e_{\ominus \alpha}(t, s), \quad t \geq s,
$$

where $C$ and $\alpha$ are positive real numbers and $\alpha$ is positive regressive, then the diagonal of the matrix $W(t, s)$ is almost periodic; that is, for any $\varepsilon>0$, there exists a relatively dense set $\Gamma$ of almost periods such that, for $r \in \Gamma$, we have

$$
\begin{array}{r}
\|W(t+r, s+r)-W(t, s)\|<\varepsilon \Gamma_{0} e_{\ominus \alpha}(t, s), \\
t, s \in \mathbb{T} \cap \mathbb{T}^{-r},
\end{array}
$$

where $\Gamma_{0}$ is a positive constant.

Proof. Since

$$
\begin{aligned}
W^{\Delta_{1}}= & A(t) W(t+r, s+r) \\
& +(A(t+r)-A(t)) W(t+r, s+r),
\end{aligned}
$$

we have

$$
\begin{aligned}
& W(t+r, s+r) \\
& =W(t, s)+\int_{s}^{t} W(t, \sigma(u))(A(t+u)-A(u)) \\
& \quad \times W(u+r, s+r) \Delta u .
\end{aligned}
$$

Further we have

$$
\begin{aligned}
& \|W(t+r, s+r)-W(t, s)\| \\
& \leq \int_{s}^{t}\|W(t, \sigma(u))\|\|A(u+r)-A(u)\| \\
& \quad \times\|W(u+r, s+r)\| \Delta u \\
& \leq \int_{s}^{t} \varepsilon K^{2} e_{\ominus \alpha}(t, \sigma(u)) \Delta u \\
& =\int_{s}^{t} \varepsilon K^{2} e_{\ominus \alpha}(t, s) e_{\ominus \alpha}(s, \sigma(u)) \Delta u \\
& \leq \varepsilon K^{2} e_{\ominus \alpha}(t, s) \int_{s}^{t} e_{\ominus \alpha}(s, \sigma(u)) \Delta u \\
& \leq \frac{\varepsilon K^{2}(1+\bar{\mu} \alpha)}{\alpha} e_{\ominus \alpha}(t, s), \quad t \geq s,
\end{aligned}
$$

where $\bar{\mu}=\sup _{t \in \mathbb{T}} \mu(t)$; then, we can get

$$
\begin{gathered}
\|W(t+r, s+r)-W(t, s)\| \\
\leq \frac{\varepsilon K^{2}(1+\bar{\mu} \alpha)}{\alpha} e_{\ominus \alpha}(t, s), \\
t, s \in \mathbb{T} \cap \mathbb{T}^{-r}, \quad t \geq s,
\end{gathered}
$$

where $\Gamma_{0}=K^{2}(1+\bar{\mu} \alpha) / \alpha$. This completes the proof.

We can prove the following theorem exactly like Theorem 38 if we let $W(t, s)=e_{\alpha}(t, s)$, so we give it straightly.

Theorem 39. For any $\varepsilon>0, \alpha \in \mathscr{R}^{+}$is positive regressive and $\alpha$ is almost periodic; then, there exists a relatively dense set $\Gamma$ of almost periods such that, for $r \in \Gamma$, we have

$$
\begin{aligned}
& \left\|e_{\ominus \alpha}(t+r, s+r)-e_{\ominus \alpha}(t, s)\right\| \\
& \quad<\varepsilon \Gamma_{0} e_{\ominus \alpha}(t, s), \quad t, s \in \mathbb{T} \cap \mathbb{T}^{-r},
\end{aligned}
$$

where $\Gamma_{0}$ is a positive constant.

Lemma 40. Let $\alpha \in \mathscr{R}^{+}$be regressive and let $\mathbb{T}$ be almost periodic; then, for any $\varepsilon>0$, there exists $\delta>0$ such that $\left|s_{1}-s_{2}\right|<\delta$ implies

$$
\left|e_{\alpha}\left(t, s_{1}\right)-e_{\alpha}\left(t, s_{2}\right)\right|<\varepsilon\left|e_{\alpha}\left(t, s_{2}\right)\right|, \quad \forall t \in \mathbb{T} .
$$

Proof. For any $s_{1}, s_{2} \in \mathbb{T}$, we have

$$
\begin{aligned}
\left|e_{\alpha}\left(t, s_{1}\right)-e_{\alpha}\left(t, s_{2}\right)\right| & =\left|e_{\alpha}\left(t, s_{2}\right) e_{\alpha}\left(s_{2}, s_{1}\right)-e_{\alpha}\left(t, s_{2}\right)\right| \\
& =\left|e_{\alpha}\left(t, s_{2}\right)\right|\left|\left(e_{\alpha}\left(s_{2}, s_{1}\right)-1\right)\right| .
\end{aligned}
$$

Since $\mathbb{T}$ is almost periodic time scale, $\mu$ is bounded on $\mathbb{T}$. Denoting that

$$
\sup _{t \in \mathbb{T}}\left|\frac{\ln (1+\mu(t) \alpha)}{\mu(t)}\right|:=C,
$$

we can take $\delta<\ln (\varepsilon+1) / C$; then,

$$
\left|e_{\alpha}\left(t, s_{2}\right)\right|\left|e_{\alpha}\left(s_{2}, s_{1}\right)-1\right|<\varepsilon\left|e_{\alpha}\left(t, s_{2}\right)\right| .
$$

This completes the proof.

We can prove the following theorem exactly like Lemma 40, so we give it straightly.

Theorem 41. Let $W(t, s)$ be the Cauchy matrix of (1) and $\alpha \epsilon$ $\mathscr{R}^{+}$is regressive. If $W(t, s)$ is continuous on $\mathbb{T} \times \mathbb{T}$, then, for any $\varepsilon>0$, there exists $\delta>0$ such that $\left|s_{1}-s_{2}\right|<\delta$ implies

$$
\left\|W\left(t, s_{1}\right)-W\left(t, s_{2}\right)\right\| \leq \varepsilon\left\|W\left(t, s_{2}\right)\right\|, \quad \forall t \in \mathbb{T} .
$$

If $W(t, s)$ satisfies

$$
\|W(t, s)\| \leq K e_{\alpha}(t, s),
$$

then $\left\|W\left(t, s_{1}\right)-W\left(t, s_{2}\right)\right\| \leq \varepsilon K e_{\alpha}\left(t, s_{2}\right)$. 
Using the above results, one can show the following theorem.

Theorem 42. If $f \in C\left(\mathbb{T} \times \mathbb{R}^{n}, \mathbb{R}^{n}\right)$ is almost periodic in $t$ uniformly for $x \in \mathbb{R}^{n}$ and the Cauchy matrix of (1) satisfies (57), $W(t, s)$ is continuous on $\mathbb{\mathbb { T }} \times \mathbb{\mathbb { T }}$ and

$$
\begin{gathered}
\|f(t, x)-f(t, y)\| \leq L\|x-y\|, \quad \forall x, y \in \mathbb{R}^{n}, \\
\frac{K L(1+\bar{\mu} \alpha)}{\alpha}<1, \quad \bar{\mu}=\sup _{t \in \mathbb{T}} \mu(t) .
\end{gathered}
$$

Then (2) has a unique continuous almost periodic solution as follows:

$$
x(t)=\int_{-\infty}^{t} W(t, \sigma(s)) f(s, x(s)) \Delta s .
$$

Proof. Let $\mathbb{D}$ be the space formed by all almost periodic functions on an almost periodic time scale $\mathbb{T}$. Define an operator $T: C\left(\mathbb{T}, \mathbb{R}^{n}\right) \rightarrow C\left(\mathbb{T}, \mathbb{R}^{n}\right)$ :

$$
T(\varphi(t))=\int_{-\infty}^{t} W(t, \sigma(s)) f(s, \varphi(s)) \Delta s .
$$

Consider the following difference:

$$
\begin{aligned}
& \|T \varphi(t+\tau)-T \varphi(t)\| \\
& =\| \int_{-\infty}^{t+\tau} W(t+\tau, \sigma(s)) f(s, \varphi(s)) \Delta s \\
& -\int_{-\infty}^{t} W(t, \sigma(s)) f(s, \varphi(s)) \Delta s \| \\
& =\| \int_{-\infty}^{t} W(t+\tau, \sigma(s+\tau)) f(s+\tau, \varphi(s+\tau)) \Delta \tau \\
& -\int_{-\infty}^{t} W(t, \sigma(s)) f(s, \varphi(s)) \Delta s \| \\
& \leq \int_{-\infty}^{t}\|W(t+\tau, \sigma(s+\tau))\| \\
& \|f(s+\tau, \varphi(s+\tau))-f(s+\tau, \varphi(s))\| \Delta s \\
& +\int_{-\infty}^{t}\|W(t+\tau, \sigma(s+\tau))-W(t, \sigma(s))\| \\
& \times\|f(s+\tau, \varphi(s))\| \Delta s \\
& +\int_{-\infty}^{t}\|W(t, \sigma(s))\| \\
& \times\|f(s+\tau, \varphi(s))-f(s, \varphi(s))\| \Delta s \\
& \leq \int_{-\infty}^{t} K L e_{\ominus \alpha}(t+\tau, \sigma(s+\tau))\|\varphi(s+\tau)-\varphi(s)\| \Delta s \\
& +\int_{-\infty}^{t} F\|W(t+\tau, \sigma(s+\tau))-W(t, \sigma(s))\| \Delta s \\
& +\int_{-\infty}^{t} K \varepsilon e_{\ominus \alpha}(t, \sigma(s)) \Delta s
\end{aligned}
$$

where $F=\sup _{(t, x) \in \mathbb{T} \times \mathbb{R}^{n}} f(t, x)$. Noting that $|\mu(t+\tau)-\mu(t)|<\varepsilon$, that is, $|\sigma(t+\tau)-(\sigma(t)+\tau)|<\varepsilon$, by Lemma 40 , we have

$$
\begin{aligned}
& \left|e_{\ominus \alpha}(t+\tau, \sigma(s+\tau))-e_{\ominus \alpha}(t+\tau, \sigma(s)+\tau)\right| \\
& \quad<\varepsilon e_{\ominus \alpha}(t+\tau, \sigma(s)+\tau)
\end{aligned}
$$

then, from (74) and Theorem 39, we have

$$
\begin{aligned}
& e_{\ominus \alpha}(t+\tau, \sigma(s+\tau)) \\
& \leq(1+\varepsilon) e_{\ominus \alpha}(t+\tau, \sigma(s)+\tau) \\
& =(1+\varepsilon)\left[e_{\ominus \alpha}(t+\tau, \sigma(s)+\tau)\right. \\
& \left.\quad-e_{\ominus \alpha}(t, \sigma(s))+e_{\ominus \alpha}(t, \sigma(s))\right] \\
& \leq\left[(1+\varepsilon) \varepsilon \Gamma_{0}+(1+\varepsilon)\right] e_{\ominus \alpha}(t, \sigma(s))
\end{aligned}
$$

and since $W(t, s)$ is continuous on $\mathbb{} \mathbb{} \times$, by Theorems 38, 39, and 41 , one has

$$
\begin{aligned}
\| W( & t+\tau, \sigma(s+\tau))-W(t, \sigma(s)) \| \\
\leq & \|W(t+\tau, \sigma(s+\tau))-W(t+\tau, \sigma(s)+\tau)\| \\
& +\|W(t+\tau, \sigma(s)+\tau)-W(t, \sigma(s))\| \\
\leq & \varepsilon K e_{\ominus \alpha}(t+\tau, \sigma(s)+\tau)+\varepsilon \Gamma_{0} e_{\ominus \alpha}(t, \sigma(s)) \\
\leq & \varepsilon K\left|e_{\ominus \alpha}(t+\tau, \sigma(s)+\tau)-e_{\ominus \alpha}(t, \sigma(s))\right| \\
& +\varepsilon\left(K+\Gamma_{0}\right) e_{\ominus \alpha}(t, \sigma(s)) \\
\leq & {\left[\varepsilon^{2} K \Gamma_{0}+\varepsilon\left(K+\Gamma_{0}\right)\right] e_{\ominus \alpha}(t, \sigma(s)), }
\end{aligned}
$$

where $\Gamma_{0}=K^{2}(1+\bar{\mu} \alpha) / \alpha$.

By (73), we can obtain

$$
\begin{aligned}
& \|T \varphi(t+\tau)-T \varphi(t)\| \\
& \leq\left\{\frac{(1+\bar{\mu} \alpha) K L\left[(1+\varepsilon)\left(\varepsilon \Gamma_{0}+1\right)\right]}{\alpha}\right. \\
& \left.\quad+\frac{F\left(\varepsilon K \Gamma_{0}+K+\Gamma_{0}\right)(1+\bar{\mu} \alpha)}{\alpha}+\frac{K(1+\bar{\mu} \alpha)}{\alpha}\right\} \varepsilon .
\end{aligned}
$$

Hence, $T \varphi(t)$ is almost periodic. Now, if $\psi, \varphi \in \mathbb{D}$, then we can obtain

$$
\begin{aligned}
& \|T \varphi(t)-T \psi(t)\| \\
& =\left\|\int_{-\infty}^{t} W(t, \sigma(s))(f(s, \varphi(s))-f(s, \psi(s))) \Delta s\right\| \\
& \leq \int_{-\infty}^{t}\|W(t, \sigma(s))\|\|f(s, \varphi(s))-f(s, \psi(s))\| \Delta s \\
& \leq \frac{K L(1+\bar{\mu} \alpha)}{\alpha}\|\varphi-\psi\| .
\end{aligned}
$$

Since $K L(1+\bar{\mu} \alpha) / \alpha<1$, then $T$ is a contraction mapping. Hence, $T$ has a fixed point in $\mathbb{D}$; that is, (2) has a unique almost periodic solution. This completes the proof. 


\section{An Application}

In the following, we present a result which can be found in [[2, Lemma 2.15] which will be essential to our purposes.

Lemma 43 (see [2]). Let $c_{i}(t)$ be an almost periodic function on $\mathbb{T}$, where $c_{i}(t)>0,-c_{i} \in \mathscr{R}, \forall t \in \mathbb{T}$, and

$$
\min _{1 \leq i \leq n}\left\{\inf _{t \in \mathbb{T}} c_{i}(t)\right\}=\widetilde{m}>0 \text {; }
$$

then, the linear system

$$
x^{\Delta}(t)=\operatorname{diag}\left(-c_{1}(t),-c_{2}(t), \ldots,-c_{n}(t)\right) x(t)
$$

admits an exponential dichotomy on $\mathbb{T}$.

Remark 44. By Remark 36, it is easy to see that one can take $W(t, \sigma(s))=X(t) X^{-1}(\sigma(s))$ and there exist positive constants $K, \alpha$ such that

$$
\|W(t, \sigma(s))\| \leq K e_{\ominus \alpha}(t, s), \quad t \geq s .
$$

That is to say, the inequality (57) in Theorem 38 holds.

Consider the following high-order Hopfield neural networks on time scales:

$$
\begin{aligned}
x_{i}^{\Delta}= & -c_{i}(t) x_{i}(t)+\sum_{j=1}^{n} a_{i j}(t) f_{j}\left(x_{j}(t)\right) \\
& +\sum_{j=1}^{n} \sum_{l=1}^{n} b_{i j l}(t) g_{j}\left(x_{j}(t)\right) g_{l}\left(x_{l}(t)\right)+I_{i}(t),
\end{aligned}
$$

for $i=1,2, \ldots, n$, where $n$ corresponds to the number of units in a neural network, $X_{i}(t)$ corresponds to the state vector of the $i$ th unite at the time $t, c_{i}(t)$ represents the rate with which the $i$ th unite will reset its potential to the resting state in isolation when disconnected from the network external inputs, $a_{i j}(t)$ and $b_{i j l}(t)$ are the first- and secondorder connection weights of neural network, $I_{i}(t)$ denotes the external inputs at time $t$, and $f_{j}$ and $g_{j}$ are the activation functions of signal transmission.

Now, we assume the following conditions are fulfilled.

$\left(H_{1}\right) x_{i}, a_{i j}, b_{i j l}, I_{i}$ are almost periodic functions, $-c_{i} \in \mathscr{R}$ and $c_{i}>0$ for every $i, j, l=1,2, \ldots, n$.

$\left(H_{2}\right)$ There exist positive constants $M_{j}, N_{j}, j=1,2, \ldots, n$ such that $\left|f_{j}(x)\right| \leq M_{j}$ and $\left|g_{j}(x)\right| \leq N_{j}$ for $j=$ $1,2, \ldots, n, x \in \mathbb{R}$.

$\left(H_{3}\right)$ Functions $f_{j}(u), g_{j}(u), j=1,2, \ldots, n$ satisfy the Lipschitz condition; that is, there exist constants $L_{j}$, $H_{j}>0$ such that $\left|f_{j}\left(u_{1}\right)-f_{j}\left(u_{2}\right)\right| \leq L_{j}\left|u_{1}-u_{2}\right|$, $\left|g_{j}\left(u_{1}\right)-g_{j}\left(u_{2}\right)\right| \leq H_{j}\left|u_{1}-u_{2}\right|, j=1,2, \ldots, n$.

$\left(H_{4}\right)$

$$
\begin{aligned}
\max _{1 \leq i \leq n}\left\{\left(\sum_{j=1}^{n} \overline{a_{i j}} L_{j}+\sum_{j=1}^{n} \sum_{l=1}^{n} \overline{b_{i j l}} N_{j} H_{l}+\sum_{j=1}^{n} \sum_{l=1}^{n} \overline{b_{i j l}} N_{l} H_{j}\right)\right. \\
\left.\times\left(\underline{c_{i}}\right)^{-1}\right\}<1,
\end{aligned}
$$

where

$$
\begin{gathered}
\underline{c_{i}}=\inf _{t \in \mathbb{T}}\left|c_{i}(t)\right|, \quad \overline{c_{i}}=\sup _{t \in \mathbb{T}}\left|c_{i}(t)\right|, \\
\overline{a_{i j}}=\sup _{t \in \mathbb{T}}\left|a_{i j}(t)\right|, \quad \overline{b_{i j l}}=\sup _{t \in \mathbb{T}}\left|b_{i j l}(t)\right|, \\
\overline{I_{i}}=\sup _{t \in \mathbb{T}}\left|I_{i}(t)\right| .
\end{gathered}
$$

Then, by hypotheses $\left(H_{1}\right),\left(H_{2}\right),\left(H_{3}\right)$, and $\left(H_{4}\right)$ and using Lemma 43 and Remark 44, we obtain that all hypotheses of Theorem 42 are satisfied; then, the system (82) possesses a unique almost periodic solution.

\section{Conclusion and Further Discussion}

In this paper, we introduce three equivalent concepts of almost periodic time scales which can strictly include the concept of periodic time scales. Several examples are given to show that there exists a class of time scales which is almost periodic but not periodic according to the new proposed definitions. Furthermore, all the results obtained in [1] are valid without considering the set $\Pi$, which will bring more generality of the obtained results in our relative previous works. Furthermore, using the almost periodicity of Cauchy matrix for dynamic equations on time scales, by fixed point theorems in Banach space, one can find some new sufficient conditions for the existence of almost periodic solutions for dynamic equations under the sense of these new definitions. Finally, all new concepts proposed in this paper will play an important and fundamental role in establishing almost periodic theory of dynamic equations on time scales.

Furthermore, according to Definition 13, one can introduce the concept of almost automorphic functions as follows.

Definition 45. Let $\mathbb{X}$ be a Banach space and let $\mathbb{T}$ be an almost periodic time scale.

(i) Let $f: \mathbb{T} \rightarrow \mathbb{X}$ be a bounded continuous function. We say that $f$ is almost automorphic if, for every sequence of real numbers $\left\{s_{n}\right\}_{n=1}^{\infty}$, we can extract a subsequence $\left\{\tau_{n}\right\}_{n=1}^{\infty}$ such that the limit set $\mathbb{T}_{0}$ of $\left\{\mathbb{T}^{-\tau_{n}}\right\}$ exists and:

$$
g(t)=\lim _{n \rightarrow \infty} f\left(t+\tau_{n}\right)
$$

is well defined for each $t \in \mathbb{T}_{0}$. Furthermore, the limit set of $\left\{\mathbb{T}_{0}^{\tau_{n}}\right\}$ is $\mathbb{T}$ and

$$
\lim _{n \rightarrow \infty} g\left(t-\tau_{n}\right)=f(t)
$$

for each $t \in \mathbb{T}$. Denote by $A A(\mathbb{T}, \mathbb{X})$ the set of all such functions.

(ii) A continuous function $f: \mathbb{T} \times B \rightarrow \mathbb{X}$ is said to be almost automorphic if $f(t, x)$ is almost automorphic in $t \in \mathbb{T}$ uniformly for all $x \in B$, where $B$ is any bounded subset of $\mathbb{X}$ or $B=\mathbb{X}$. Denote by $A A(\mathbb{T} \times$ $\mathbb{X}, \mathbb{X})$ the set of all such functions. 
Under Definition 45, all the obtained results and proof process in [3] are valid and this new kind of time scales proposed in this paper will bring more general sense to our future research works.

Remark 46. In Definition 45, if $t \in \mathbb{T}_{0}$, then $t+\tau_{n} \in \mathbb{T}_{0}^{\tau_{n}}=$ $\left(\lim _{n \rightarrow \infty} \mathbb{T}^{-\tau_{n}}\right)^{\tau_{n}}=\mathbb{T}$. Similarly, if $t \in \mathbb{T}$, then $t-\tau_{n} \in \mathbb{T}^{-\tau_{n}}=$ $\left(\lim _{n \rightarrow \infty} \mathbb{T}_{0}^{\tau_{n}}\right)^{-\tau_{n}}=\mathbb{T}_{0}$. Hence, the (i) of Definition 45 makes sense.

\section{Conflict of Interests}

The authors declare that they have no competing interests.

\section{Authors' Contribution}

All authors contributed equally to the paper and typed, read, and approved the final paper.

\section{Acknowledgments}

The authors would like to express their sincere thanks to the editor Professor Dr. Elena Braverman for handling our paper during the reviewing process and to the referees for suggesting some corrections that help making the content of the paper more accurate. This work is supported by the foundation of Yunnan University in China (no. 2013CG020).

\section{References}

[1] Y. Li and C. Wang, "Uniformly almost periodic functions and almost periodic solutions to dynamic equations on time scales," Abstract and Applied Analysis, vol. 2011, Article ID 341520, 22 pages, 2011.

[2] Y. Li and C. Wang, "Almost periodic functions on time scales and applications," Discrete Dynamics in Nature and Society, vol. 2011, Article ID 727068, 20 pages, 2011.

[3] C. Wang and Y. Li, "Weighted pseudo almost automorphic functions with applications to abstract dynamic equations on time scales," Annales Polonici Mathematici, vol. 108, no. 3, pp. 225-240, 2013.

[4] C. Wang and R. P. Agarwal, "Weighted piecewise pseudo almost automorphic functions with applications to abstract impulsive $\nabla$-dynamic equations on time scales," Advances in Difference Equations, vol. 2014, article 153, 2014.

[5] C. Wang, "Almost periodic solutions of impulsive BAM neural networks with variable delays on time scales," Communications in Nonlinear Science and Numerical Simulation, vol. 19, no. 8, pp. 2828-2842, 2014.

[6] Y. Li and C. Wang, "Pseudo almost periodic functions and pseudo almost periodic solutions to dynamic equations on time scales," Advances in Difference Equations, vol. 2012, article 77, 2012.

[7] C. Lizama and J. G. Mesquita, "Almost automorphic solutions of dynamic equations on time scales," Journal of Functional Analysis, vol. 265, no. 10, pp. 2267-2311, 2013.

[8] J. Alzabut, Y. Bolat, and T. Abdeljawad, "Almost periodic dynamics of a discrete Nicholson's blowflies model involving a linear harvesting term," Advances in Difference Equations, vol. 2012, article 158, 2012.
[9] J. O. Alzabut, "Existence and exponential convergence of almost periodic solutions for a discrete Nicholson's Blowflies model with nonlinear harvesting term," Mathematical Sciences Letters, vol. 2, no. 3, pp. 201-207, 2013.

[10] Y. Guan and K. Wang, "Translation properties of time scales and almost periodic functions," Mathematical and Computer Modelling, vol. 57, no. 5-6, pp. 1165-1174, 2013.

[11] T. Liang, Y. Yang, Y. Liu, and L. Li, "Existence and global exponential stability of almost periodic solutions to CohenGrossberg neural networks with distributed delays on time scales," Neurocomputing, vol. 123, pp. 207-215, 2014.

[12] Y. Wang and L. Li, "Almost periodic solutions for second order dynamic equations on time scales," Discrete Dynamics in Nature and Society, vol. 2013, Article ID 784198, 11 pages, 2013.

[13] C. Lizama, J. G. Mesquita, and R. Ponce, "A connection between almost periodic functions defined on timescales and $\mathbb{R}$, , Applicable Analysis, 2014.

[14] C. Lizama and J. G. Mesquita, "Asymptotically almost automorphic solutions of dynamic equations on time scales," Journal of Mathematical Analysis and Applications, vol. 407, pp. 339-349, 2013.

[15] E. R. Kaufmann and Y. N. Raffoul, "Periodic solutions for a neutral nonlinear dynamical equation on a time scale," Journal of Mathematical Analysis and Applications, vol. 319, no. 1, pp. 315-325, 2006.

[16] B. Jackson, "Partial dynamic equations on time scales," Journal of Computational and Applied Mathematics, vol. 186, no. 2, pp. 391-415, 2006.

[17] M. Bohner and A. Peterson, Dynamic Equations on Time Scales: An Introduction with Applications, Birkhäuser, Boston, Mass, USA, 2001.

[18] R. Agarwal, M. Bohner, D. O'Regan, and A. Peterson, "Dynamic equations on time scales: a survey," Journal of Computational and Applied Mathematics, vol. 141, no. 1-2, pp. 1-26, 2002.

[19] A. M. Fink, Almost Periodic Differential Equations, Springer, Berlin, Germany, 1974. 


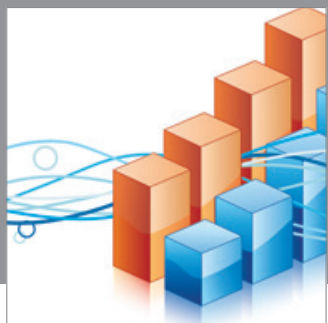

Advances in

Operations Research

mansans

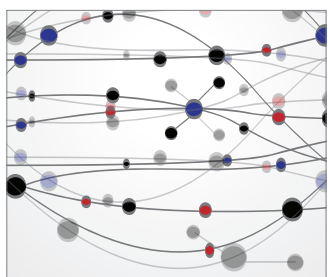

The Scientific World Journal
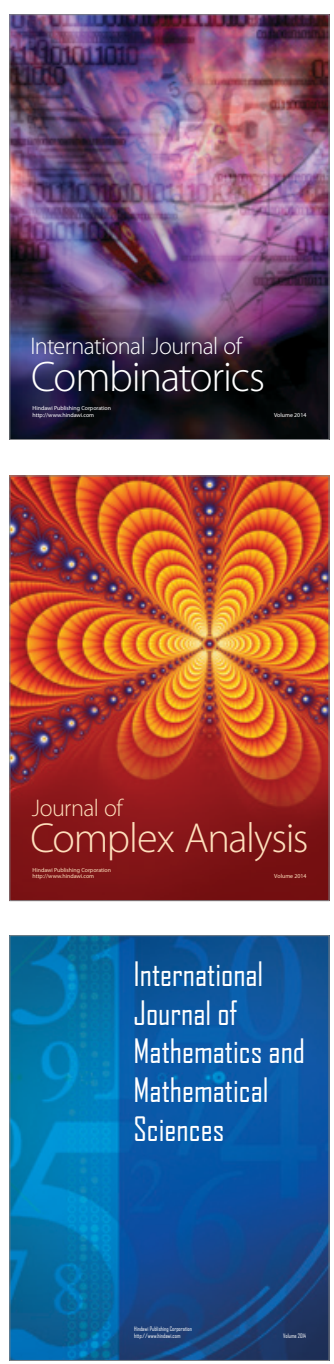
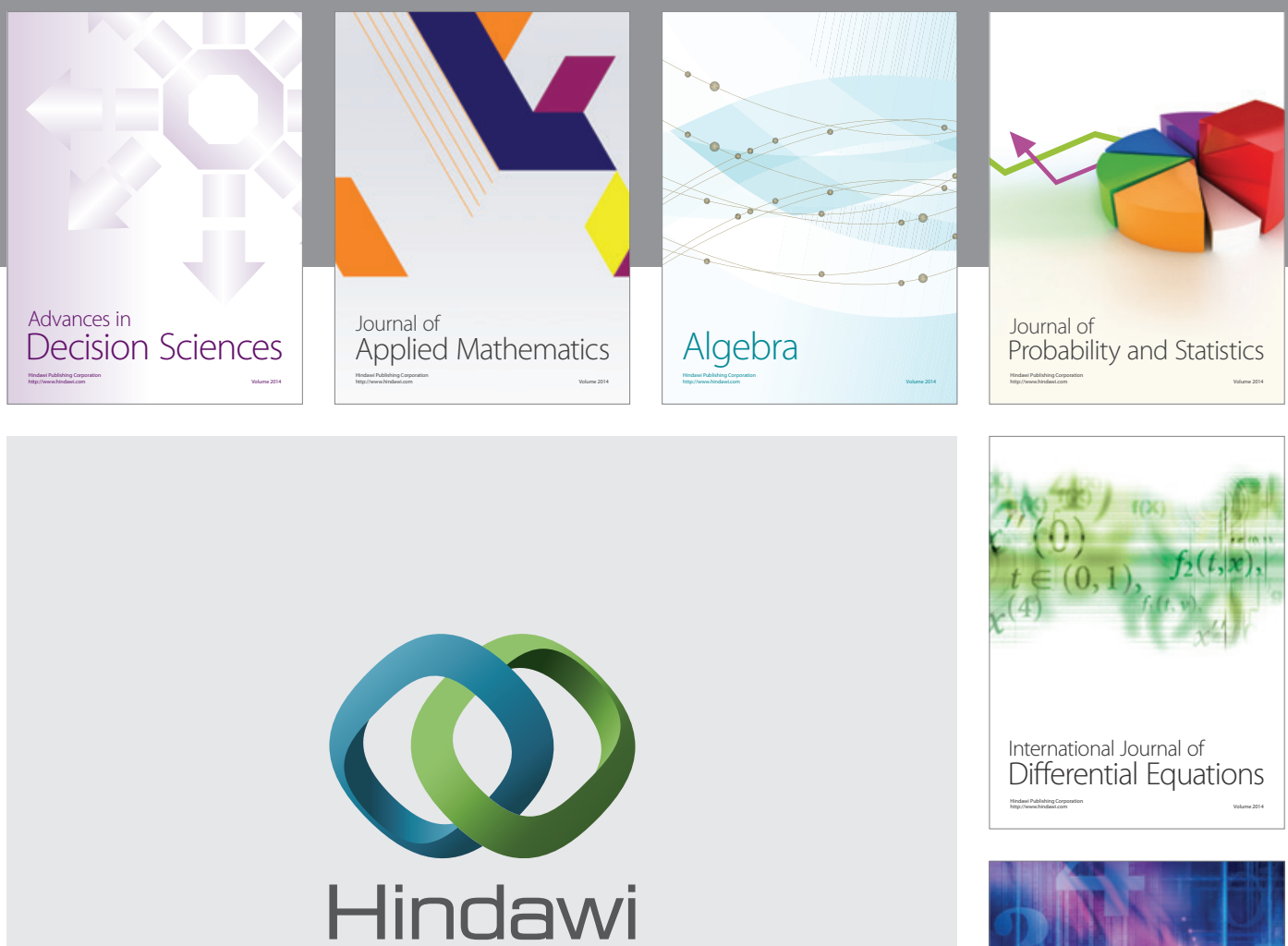

Submit your manuscripts at http://www.hindawi.com
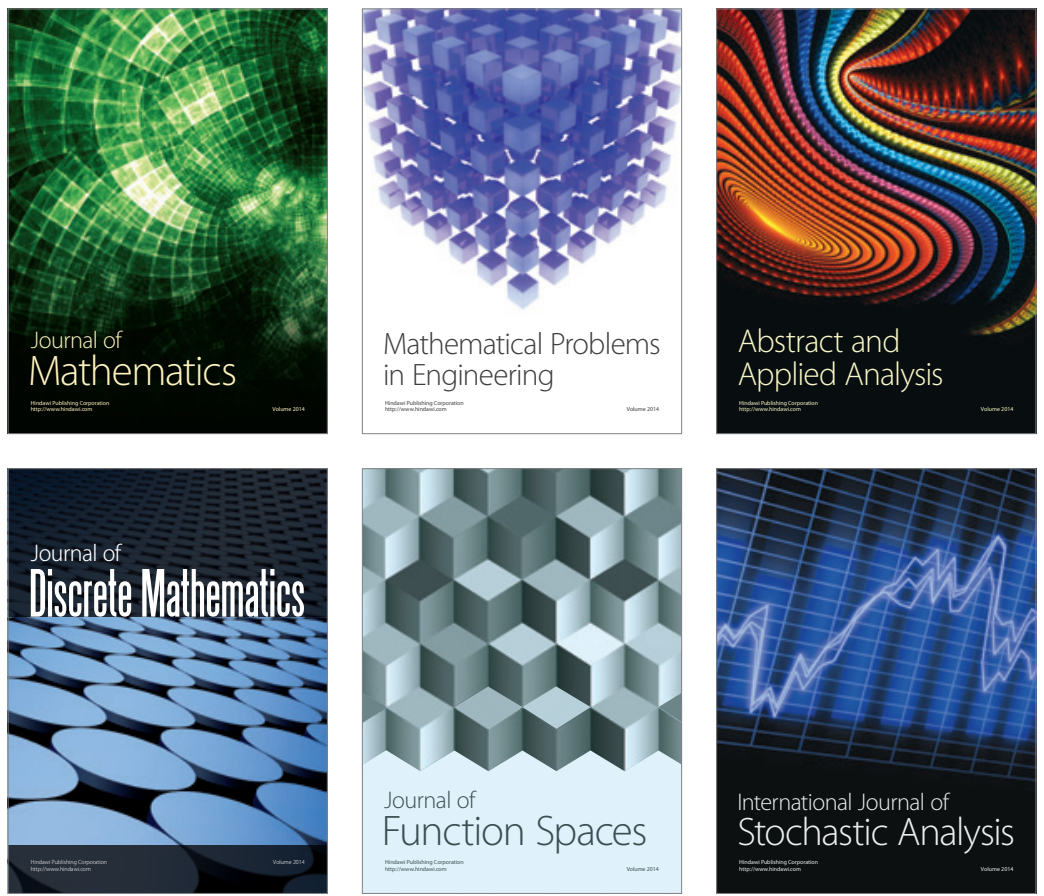

Journal of

Function Spaces

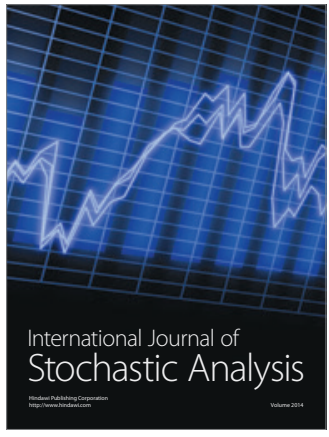

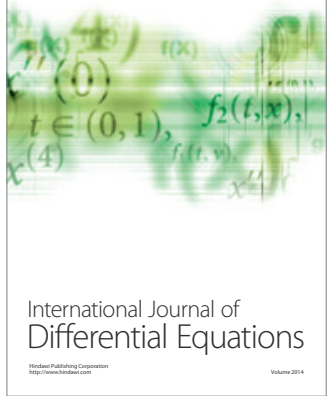
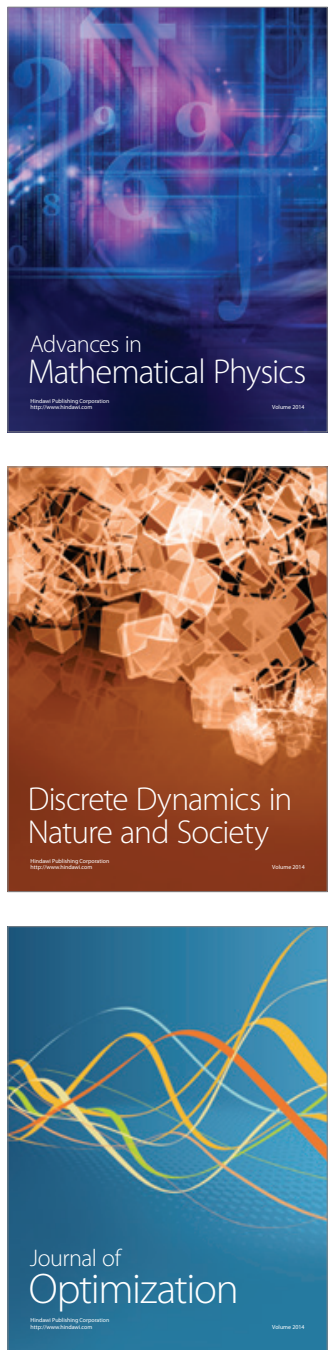